\title{
Measurement and Analytical Modeling of the $\Delta E$ Effect in a Bulk Iron-Cobalt Alloy
}

\author{
Olivier Hubert ${ }^{1}$ and Laurent Daniel ${ }^{2}$ \\ ${ }^{1}$ LMT-Cachan, ENS-Cachan/CNRS/UPMC/UniverSud Paris, F-94235 Cachan cedex, France \\ ${ }^{2}$ LGEP, Supelec/CNRS/Univ Paris-Sud/UPMC, F-91192 Gif-sur-Yvette Cedex, France
}

\begin{abstract}
The Young modulus $E$ of a material is the ratio between an uniaxial applied stress and the elastic strain measured in the direction parallel to the applied stress. For magnetic materials, stress-strain relationship appears to be non-linear. This effect is called the $\Delta E$ effect, usually introduced as a dependency of the Young modulus on the stress level, but physically due to a re-organization of the magnetic domains under stress generating a magnetostriction strain. In this paper, the $\Delta E$ effect is measured for a bulk iron-cobalt alloy thanks to two different methods. An analytical magneto-mechanical modeling of the $\Delta E$ effect is finally applied and compared to the experimental results.
\end{abstract}

Index Terms-Analytical modeling, Delta E effect, magnetoelasticity.

\section{INTRODUCTION}

$\mathbf{T}$ HE YOUNG modulus $E$ of a material is the ratio between stress $\sigma$ and longitudinal strain $\varepsilon / /$ when a tension or compression test is performed in elastic condition ((1)).

$$
E=\frac{\sigma}{\varepsilon_{/ /}}
$$

When a stress is applied to a magnetic material, stress-strain response appears to be non-linear (Fig. 1). This effect is called the $\Delta E$ effect [1], [2]. It is often introduced and discussed as a dependency of the Young modulus $E$ on the stress level. On the other hand, the $\Delta E$ effect depends on the state of magnetization of the material: the Young modulus of a demagnetized specimen appears to be lower (by an amount $\Delta E$ ) than the Young modulus of the same specimen magnetized at saturation. Thus, the $\Delta E$ effect can be seen as an apparent loss of linearity of the elastic behavior of demagnetized specimens. But it can also be interpreted as a consequence of the effect of stress on the magnetostriction strain. The $\Delta E$ effect can consequently be dissociated from the elastic behavior.

In the first part of this paper, the $\Delta E$ effect is defined as a function of the magnetostriction strain under stress. In Sections IV and V, two distinct methods are proposed to measure the $\Delta E$ effect. These methods are applied to an iron-cobalt alloy presented in part III. Finally, these experimental results are compared to the results of a semi-analytical model recently proposed [3].

\section{DEFINITION OF THE $\Delta E$ EFFECT}

Magnetostriction $\varepsilon^{\mu}$ is usually observed during the magnetization process of a magnetic material [2]. It is the consequence of the change of the domain structure. The magnetic microstructure can also be modified by application of a stress $\sigma$. This stress

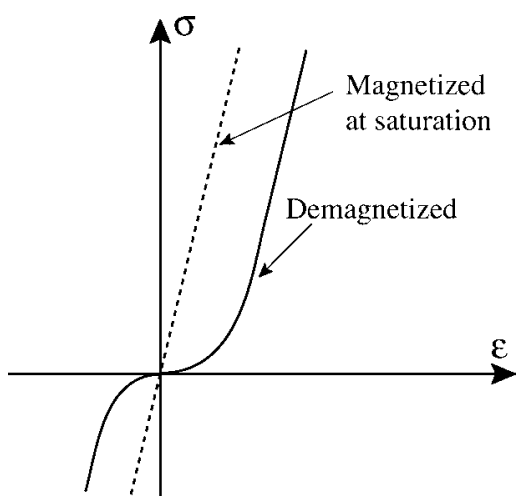

Fig. 1. Illustration of $\Delta E$ effect on a tensile stress-strain curve [1].

generates a magnetostriction strain $\boldsymbol{\varepsilon}^{\mu}$ as well. During a tension or compression test on a magnetic specimen, the magnetostriction strain $\varepsilon^{\mu}$ is superimposed to the elastic strain $\varepsilon^{e l}$, so that the total measured strain $\varepsilon$ is defined by (2). The apparent Young modulus $E_{a}$ is then defined by (3) $\left(\varepsilon_{/ /}^{e l}\right.$ and $\varepsilon_{/ /}^{\mu}$ are the longitudinal components of $\varepsilon^{e l}$ and $\varepsilon^{\mu}$ ).

$$
\begin{aligned}
\varepsilon & =\varepsilon^{e l}+\varepsilon^{\mu} \\
E_{a} & =\frac{\sigma}{\varepsilon_{/ /}^{e l}+\varepsilon_{/ /}^{\mu}}
\end{aligned}
$$

In the case of a strongly magnetized material, the domain structure has reached a saturated configuration: the magnetostriction strain cannot evolve anymore. The apparent Young modulus $E_{a}^{s}$ is then defined by (4), corresponding to the original definition of the Young modulus given by (1) in absence of magnetostriction.

$$
E_{a}^{s}=\frac{\sigma}{\varepsilon_{/ /}^{e l}}
$$

For a given initial magnetic configuration, the $\Delta E$ effect can be quantitatively defined as a function of the uniaxial applied stress $\sigma$ following equation (5).

$$
\frac{\Delta E}{E}=\frac{E_{a}^{s}-E_{a}(\sigma)}{E(\sigma)}=\frac{\varepsilon_{/ /}^{\mu}(\sigma)}{\varepsilon^{e l}(\sigma)}
$$


The $\Delta E$ effect can then be fully described if the evolution of magnetostriction with respect to stress is given. In this paper we follow this approach for an iron-cobalt alloy.

\section{MATERial PRESENTATION}

The material is a $49 \% \mathrm{Co}-49 \% \mathrm{Fe}-2 \% \mathrm{~V}$ alloy (body-cubiccentered crystallographic structure), delivered in $2.5 \mathrm{~mm}$ thick, $110 \mathrm{~mm}$ long and $12.5 \mathrm{~mm}$ wide bands (from Imphy Alloys). Cobalt based alloys are usually known to exhibit a strong saturation magnetization $\left(M_{s}=1.91 \times 10^{6} \mathrm{~A} / \mathrm{m}\right)$, leading to high torque/weigth performance for aeronautic equipments. This alloy exhibits a low value of magnetocrystalline constant $K_{1}\left(K_{1}<2 \mathrm{~kJ} . \mathrm{m}^{-3}\right)$ that leads to a reduced influence of the crystallographic texture. The magnetostriction constants $\lambda_{100}$ and $\lambda_{111}$ are positive and high $\left(\lambda_{100}=12 \times 10^{-5}\right.$, $\lambda_{111}=4 \times 10^{-5}$ ). These high values induce a high sensitivity of the magnetic behavior to stress.

\section{Measurement of $\Delta E$ EFFect From Stress-Strain MEASUREMENTS: METHOD 1}

\section{A. Basic Stress-Strain Behavior of Iron-Cobalt Alloy}

The measurement of $\Delta E$ effect is usually based on the evaluation of the stress-strain response of a demagnetized specimen thanks to a tensile-compressive machine. Fig. 2 shows the typical stress-strain behavior obtained with the iron-cobalt alloy. Strain is measured thanks to bidirectional strain gages stuck on the two sides of the sample. A force cell allows to measure the stress value (quasistatic alternative tensile-compressive experiments). The full and dashed lines respectively correspond to the longitudinal and transverse total strain $\varepsilon_{/ /}$and $\varepsilon_{\perp}$. The $\Delta E$ effect results in a strong non-linearity close to zero stress, followed by linear portions of curves at higher stress level (in tension or compression). A linear interpolation on the high stress linear parts of the longitudinal curve allows the estimation of the Young modulus $E$. The Poisson ratio $\nu$ can be estimated as well using the transverse curve ((6)). We obtain $E \approx 235 \mathrm{GPa}$ and $\nu \approx 0.27$. The magnetostrictive part of the total deformation can then be extracted from the total deformation according to (2) and (4).

$$
\nu=-\varepsilon_{\perp}^{e l} / \varepsilon_{/ /}^{e l}
$$

\section{B. Extraction of the $\Delta E$ Effect}

Magnetostriction strain versus stress behavior can be extracted from the experiments. Combining (2) and elastic constitutive law, we obtain the definition of longitudinal and transverse magnetostriction strain $\left(\varepsilon_{/ /}^{\mu}\right.$ and $\left.\varepsilon_{\perp}^{\mu}\right)$ depending on the measured longitudinal and transverse components $(\varepsilon / /$ and $\varepsilon_{\perp}$ ) of the total strain $\varepsilon$.

$$
\begin{aligned}
& \varepsilon_{/ /}^{\mu}(\sigma)=\varepsilon_{/ /}(\sigma)-\varepsilon_{/ /}^{e l}(\sigma)=\varepsilon_{/ /}(\sigma)-\sigma / E \\
& \varepsilon_{\perp}^{\mu}(\sigma)=\varepsilon_{\perp}(\sigma)-\varepsilon_{\perp}^{e l}(\sigma)=\varepsilon_{\perp}(\sigma)+(\nu \sigma) / E
\end{aligned}
$$

The results for the mean values of $\varepsilon_{/ /}^{\mu}(\sigma)$ and $\varepsilon_{\perp}^{\mu}(\sigma)$ are reported in Fig. 6 (full lines). $\varepsilon_{/ /}^{\mu}(\sigma)$ is clearly non-linear and

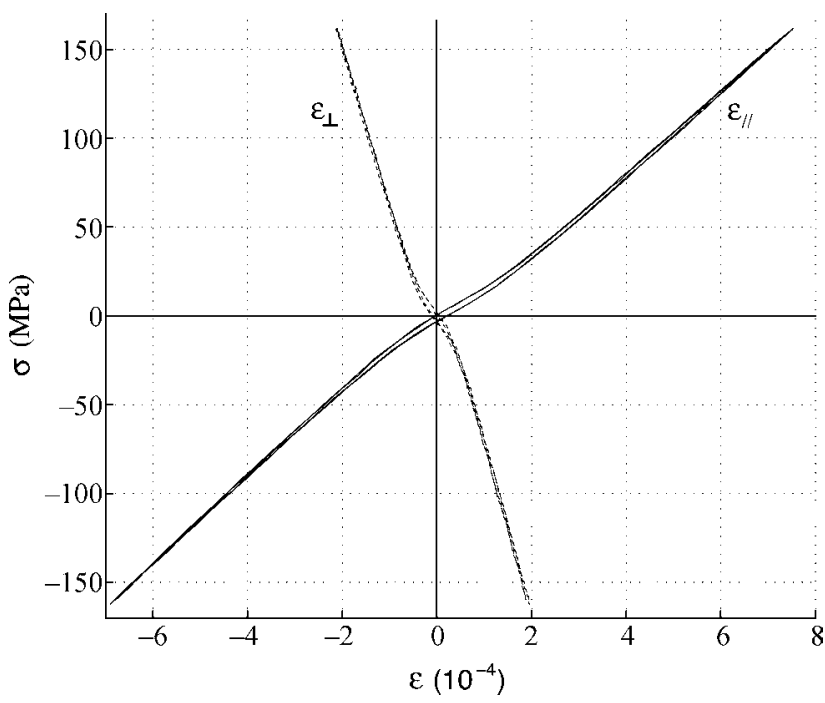

Fig. 2. Experimental stress-strain curve: longitudinal and transverse strain.

tends to saturate for high stress values (tension and compression). We observe that strain magnitude is twice lower in compression than in tension, and twice lower for the transverse measurement compared to the longitudinal one.

\section{MEASUREMENT of $\Delta E$ EFFeCt From Magnetostriction MEASUREMENTS: METHOD}

In a classical tension-compression test, the magnitude of magnetostriction is most of the time much lower than the total deformation $\varepsilon$, leading to a high level of uncertainty in the magnetostriction measurement. An alternative procedure has been recently proposed to measure the $\Delta E$ effect [3]. This procedure, based on the uniqueness of the magnetic domain configuration at magnetic saturation, is explained hereafter.

\section{A. Principle}

The procedure is based on anhysteretic magnetostriction measurements as a function of the magnetization $M$ under different levels of applied stress $\sigma_{0}$. Stress application and demagnetization procedure create an initial deformation $\varepsilon_{i}$ of the material that cannot easily be measured, but corresponds to the sum of an elastic and a magnetostrictive deformation ((9)).

$$
\varepsilon_{i}\left(M=0, \sigma_{0}\right)=\varepsilon^{e l}\left(\sigma_{0}\right)+\varepsilon^{\mu}\left(M=0, \sigma_{0}\right)
$$

Before the signal acquisition, this deformation is arbitrarily put to zero. An anhysteretic magnetostriction measurement is then performed [4]. The measured strain $\varepsilon^{\prime}$ is the variation of deformation due to magnetization at constant stress. Because elastic deformation is now unchanged (constant stress), $\varepsilon^{\prime}\left(M, \sigma_{0}\right)$ can be expressed only as a variation of magnetostrictive deformation:

$$
\begin{aligned}
\varepsilon^{\prime}\left(M, \sigma_{0}\right) & =\varepsilon\left(M, \sigma_{0}\right)-\varepsilon_{i}\left(M=0, \sigma_{0}\right) \\
& =\varepsilon^{\mu}\left(M, \sigma_{0}\right)-\varepsilon^{\mu}\left(M=0, \sigma_{0}\right)
\end{aligned}
$$

Fig. 3 gives a schematic view of $\varepsilon_{/ /}^{\prime}\left(M, \sigma_{0}\right)$ for $\sigma_{0}=0$ (a) and $\sigma_{0} \neq 0$ (b). Because of the experimental procedure, $\varepsilon_{/ /}^{\prime}\left(0, \sigma_{0}\right)$ is always zero whatever the stress level. As illustrated 

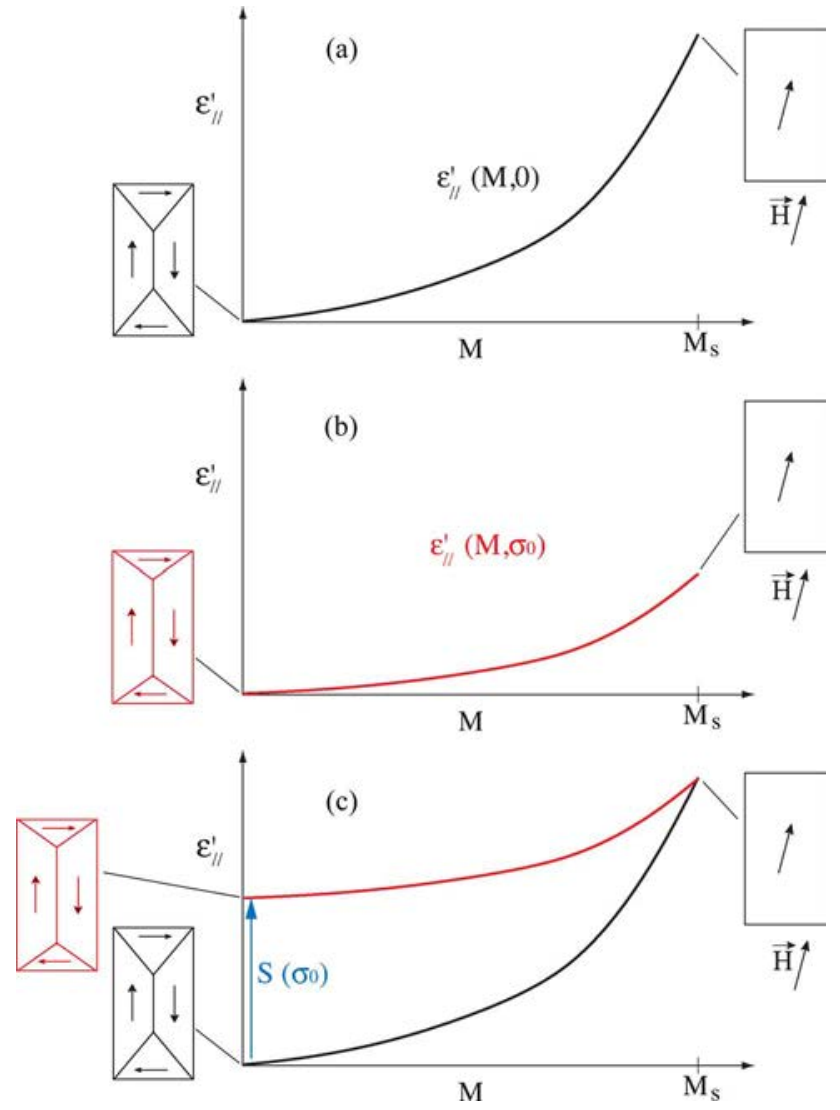

Fig. 3. Schematic view of the measured deformation $\varepsilon^{\prime},(M)$ and associated domain structure: (a) under no applied stress $\left(\sigma_{0}=0\right)$, (b) under applied stress $\left(\sigma_{0} \neq 0\right)$, (c) shift of the $\sigma_{0}$ curve to get the same value of magnetostriction at magnetic saturation.

by the very simple 2-D scheme of domains distribution in a single crystal, the domain configuration at saturation is assumed to be identical [4] (magnetization parallel to the magnetic field direction), whatever the level of stress $\sigma_{0}$. The magnetostriction at saturation should then be the same whatever the stress level. The saturation value $\varepsilon_{/ /}^{\prime}\left(M_{s}, 0\right)$ under no applied stress, equal to $\varepsilon_{/ /}^{\mu}\left(M_{s}, 0\right)$, is taken as the reference saturation value. All the $\varepsilon_{/ /}^{\prime}\left(M, \sigma_{0}\right)$ curves must reach this saturation point. We proceed to the corresponding shift $S\left(\sigma_{0}\right)$ of the $\varepsilon_{/ /}^{\prime}\left(M, \sigma_{0}\right)$ curves (Fig. 3(c)). $S\left(\sigma_{0}\right)$ thus defines the magnetostriction at zero applied field $\varepsilon_{/ /}^{\mu}\left(0, \sigma_{0}\right)$, that theoretically defines a point of the $\varepsilon_{/ /}^{\mu}(\sigma)$ curve.

\section{B. Experimental Procedure}

The benchmark for magneto-mechanical measurements (Fig. 4) is constituted of two face-to-face positioned ferrimagnetic U-yokes, between which a sample is placed. Samples have been instrumented with longitudinal and transverse strain gages stuck on both sides and a pick-up coil for the measurement of magnetic induction. A primary winding is placed on the specimen. A H-coil allows the measurement of magnetic field. The measurement is restricted to anhysteretic behavior [4]: the anhysteretic curves are measured point after point by
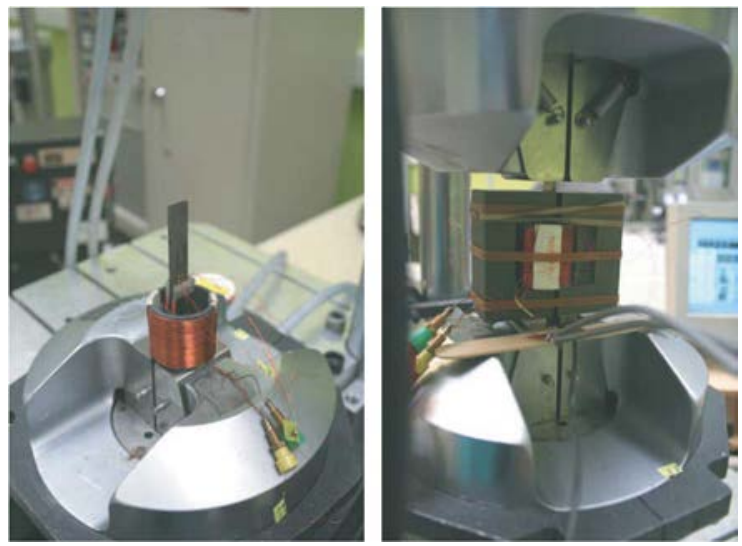

Fig. 4. Apparatus for measurement of magnetostriction under stress.

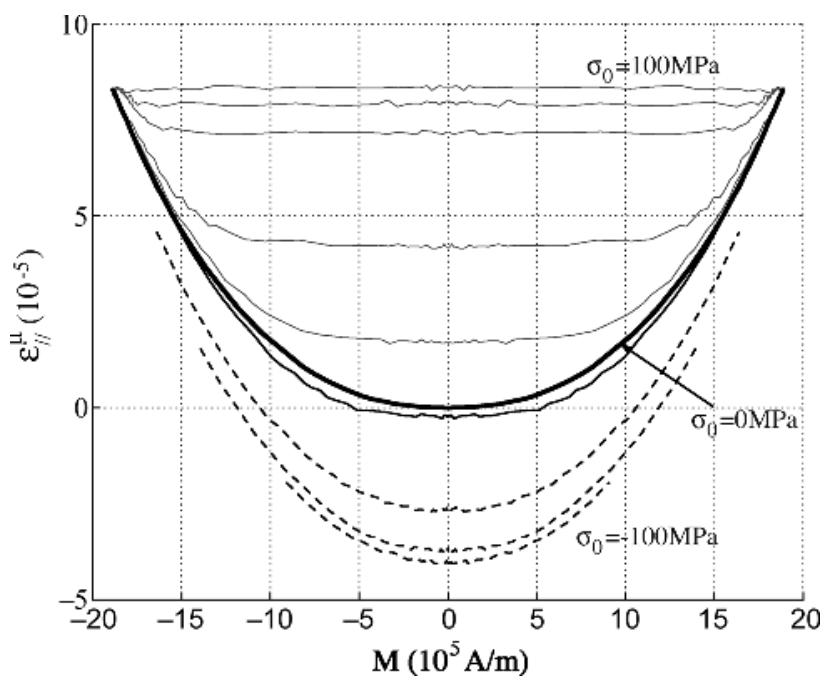

Fig. 5. Influence of uniaxial stress on the anhysteretic longitudinal magnetostriction of iron-cobalt—dashed lines: extrapolated shift.

applying a sinusoidal magnetic field of mean value $H$, and of exponentially decreasing amplitude.

Apparatus and sample are placed between the hydraulic jaws of a tensile-compressive machine (Fig. 4). The longitudinal magnetostrictive behavior of the iron-cobalt alloy under stress is reported in Fig. 5.

The procedure detailed in Section V-A is then applied. The results for the longitudinal and transverse magnetostriction as a function of stress have been reported in Fig. 6 (square dots). We observe that they are in very good agreement with the results obtained using the stress-strain curve method (method 1). Next section is dedicated to the modeling of this behavior.

\section{ANalytical Modeling of the $\Delta E$ EFfect}

An analytical modeling for the $\Delta E$ effect in magnetic materials has recently been proposed [3]. It is based on the description of the effect of stress on the magnetostriction strain. The polycrystal is seen as an aggregate of magnetic domains with random orientation. The magnetostriction $\varepsilon_{\alpha}^{\mu}$ of the corresponding domain is approximated by (11) in the coordinate 


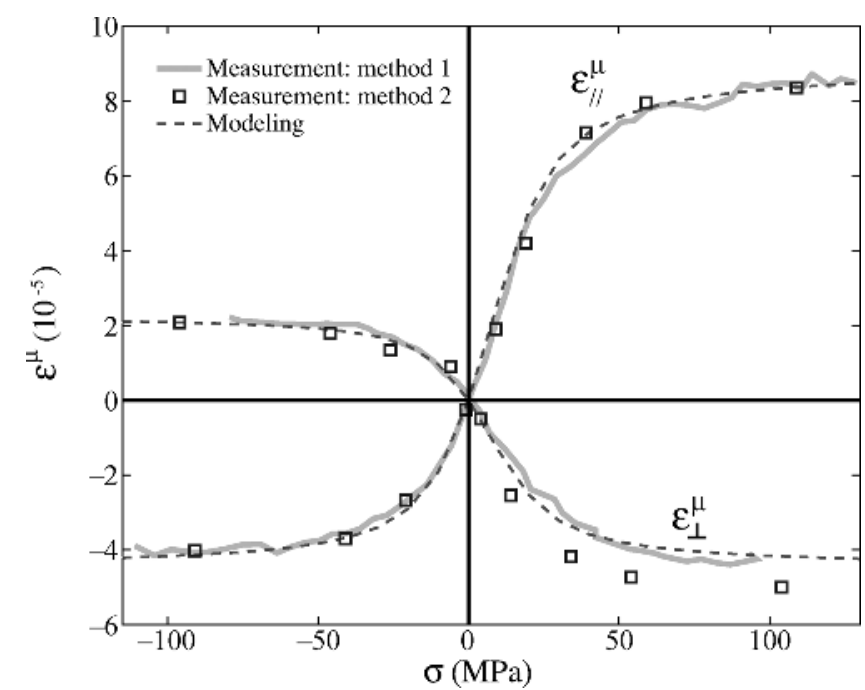

Fig. 6. $\Delta E$ effect for $49 \% \mathrm{Co}-49 \% \mathrm{Fe}-2 \% \mathrm{~V}$ alloy: longitudinal and transverse magnetostriction strain as a function of the applied stress $\sigma$. Experimental and modeling results.

system associated with the considered magnetic domain, direction 1 being the direction of the magnetization in the domain.

$$
\varepsilon_{\alpha}^{\mu}=\frac{1}{2} \lambda_{\mathrm{m}}\left(\begin{array}{ccc}
2 & 0 & 0 \\
0 & -1 & 0 \\
0 & 0 & -1
\end{array}\right)
$$

$\lambda_{\mathrm{m}}$ denotes, for the polycrystal, the maximum magnetostriction strain that can be reached under a mechanical loading. Under such hypotheses, and considering a uniaxial stress $\sigma$, the macroscopic magnetostriction strain tensor can be written: ${ }^{1}$

$$
\varepsilon^{\mu}=\frac{\pi \lambda_{\mathrm{m}} I_{1}}{2 S} \exp \left(\frac{1}{2} A_{s} \lambda_{\mathrm{m}} \sigma\right)\left(\begin{array}{ccc}
-1 & 0 & 0 \\
0 & -1 & 0 \\
0 & 0 & 2
\end{array}\right)
$$

with

$$
S=2 \pi \mathrm{e}^{\left(-\frac{1}{2} A_{s} \lambda_{\mathrm{m}} \sigma\right)} \int_{0}^{\pi} \exp \left(\frac{3}{2} A_{s} \lambda_{\mathrm{m}} \sigma \cos ^{2} \varphi\right) \sin \varphi d \varphi
$$

and

$$
I_{1}=\int_{0}^{\pi}\left(3 \cos ^{2} \varphi-1\right) \exp \left(\frac{3}{2} A_{s} \lambda_{\mathrm{m}} \sigma \cos ^{2} \varphi\right) \sin \varphi d \varphi
$$

$A_{S}$ is a material parameter linked to the initial anhysteretic susceptibility $\chi_{o}$ and to the saturation magnetization $M_{s}((15))$ already introduced in [5]. In the case of the iron-cobalt alloy studied in this paper, we used $A_{s}=1.510^{-3} \mathrm{~m}^{3} / \mathrm{J}$.

$$
A_{s}=\frac{3 \chi_{o}}{\mu_{o} M_{s}^{2}}
$$

${ }^{1}$ Full calculation and hypotheses are detailed in [3].
The definition of $\lambda_{m}$ is not simple since it is the result of both wall displacement and magnetization rotation. When only wall displacement is considered (no rotation), $\lambda_{m}$ is associated to the saturation magnetostriction with magnetization kept along easy axes. $\lambda_{m}$ can then be defined from the value of the magnetostriction coefficient $\lambda_{100}$ or $\lambda_{111}$ of the single crystal. It is shown in [3], [5] that the maximum magnetostriction strain $\lambda_{m}$ of a polycrystal with positive $K_{1}$ under such conditions can be written in the form: $\lambda_{\mathrm{m}}=(2 / 5) \lambda_{100}$. The application of this relation would lead to the value $5 \times 10^{-5}$ for $\lambda_{\mathrm{m}}$, that is a too low value compared to experimental results. This discrepancy is the signature that rotation occurs even at low stress for the iron-cobalt alloy. Nevertheless an analytical expression of $\lambda_{\mathrm{m}}$ taking rotation into account is much more difficult to obtain. The direction of magnetization is the result of the minimization of the free energy of the domains (namely magnetocrystalline and magnetoelastic energies): it does generally not correspond to the direction of the applied stress. $\lambda_{\mathrm{m}}$ consequently differs from the usual $\lambda_{s i}=(2 / 5) \lambda_{100}+(3 / 5) \lambda_{111}$ saturation value obtained under magnetic field [1], [5]. A trivial exact solution exists for isotropic magnetostriction $\left(\lambda_{100}=\lambda_{111}\right): \lambda_{\mathrm{m}}=\lambda_{100}=\lambda_{s i}$. In the present study, an optimization of $\lambda_{\mathrm{m}}$ to $\lambda_{\mathrm{m}}=9.0 \times 10^{-5}$ allows to fit properly the experimental results (Fig. 6-dashed lines: modeling).

\section{CONCLUSION}

The $\Delta E$ effect has been studied for an iron-cobalt alloy $(49 \% \mathrm{Co}-49 \% \mathrm{Fe}-2 \% \mathrm{~V})$. Two methods have been used for its experimental measurement and gave similar results. The first method is based on usual uniaxial stress-strain measurements, and the second one is based on the measurement of magnetostriction under magnetic field for several constant applied stresses. A two-parameter modeling has been used to reproduce these experimental results, with very satisfying agreement if magnetization rotation is not neglected in the definition of the stress saturated magnetostriction. This model provides a simple tool to describe the effect of stress on the magnetostriction strain. It could be used in electrical engineering to improve the macroscopic models for magneto-elastic coupling, that often neglect the effect of stress on magnetostriction.

\section{REFERENCES}

[1] B. D. Cullity, Introduction to Magnetic Materials. Reading, MA: Addison-Wesley, 1972

[2] E. Du Trémolet de Lacheisserie, Magnetostriction, Theory and Applications of Magnetoelasticity. Boca Raton, FL: CRCPress, 1993.

[3] L. Daniel and O. Hubert, "An analytical model for the DeltaE effect in magnetic materials," EPJ Appl. Phys., vol. 45, p. 31101, 2009.

[4] O. Hubert, L. Daniel, and R. Billardon, "Experimental analysis of the magnetoelastic anisotropy of a non-oriented silicon iron alloy," $J$. Magn. Magn. Mater., vol. 254-255, pp. 352-354, 2003.

[5] L. Daniel, O. Hubert, N. Buiron, and R. Billardon, "Reversible magneto-elastic behavior: A multiscale approach," J. Mech. Phys. Solids, vol. 56, pp. 1018-1042, 2008. 